\title{
Whole Genome Sequencing in single CTC improves clinical outcome in Her-2 negative breast cancer patients
}

\section{Yongping Li}

Shanghai Pudong Hospital

Hao Yuan

Shanghai Pudong Hospital

Bin Zhang

Yulin City First Hospital Yulin Branch

Xiaofei Jiang

Shanghai Pudong Hospital

Minghua Yu

Shanghai Pudong Hospital

Hongbo Zhu

Shanghai Pudong Hospital

Qinghua You

Shanghai Pudong Hospital

\section{Ling Wang}

Xijing Hospital

Bo Yu ( $\sim$ surgery_tumor@163.com )

Shanghai Pudong Hospital https://orcid.org/0000-0003-3888-9901

\section{Research article}

Keywords: Single Circulating Tumor Cell, Breast Cancer, Heterogeneity, Whole Genome Sequencing (WGS), HER2 (ERBB2)

Posted Date: October 30th, 2020

DOI: https://doi.org/10.21203/rs.3.rs-97189/v1

License: (1) (1) This work is licensed under a Creative Commons Attribution 4.0 International License.

Read Full License 


\section{Abstract}

Backgroud: Tumor tissues are usually highly heterogeneous and difficult to characterize which could mislead treatment strategy. Circulating tumor cells (CTCs) represent the most active and invasive tumor cells. This study retrospectively investigated the feasibility of individualized treatment of breast cancer patients based on genome sequencing of single cell CTC. Twenty-four CTCs were identified in three patients with breast cancer. For each patient, one polyploid CTC was captured and on which the whole genome sequencing (WGS) was performed. Based on the histopathological Her-2 status in tumor tissue and the HER2 gene status in WGS results of CTC, we adjusted treatment strategies, and monitored disease progression.

Results: Patient ID1 and ID2 are with Her-2 protein overexpression in primary tumors and HER2 gene amplification in the DNA of CTCs. In patient ID3, histological examination of primary tumor and liver metastases revealed Her-2 negative, but the WGS analysis of CTC showed that the HER2 gene was amplified. After adjusting treatment by adding Her-2 inhibitors according to the results of CTC sequencing, the liver metastases and pleural effusion were significantly reduced 2 month later, CTC number and ctDNA burden were decreased, and 18-month progression-free survival (PFS) was recorded. In addition, some potential therapeutic targets and mutations in drug-resistant genes were found.

Conclusions: The results of CTC sequencing effectively guided treatment of a patient with HER2 gene amplification in CTC but with Her-2 negative on tumor tissue. CTC sequencing is useful in resolving the heterogeneity of tumors and providing precision medicine for patients.

\section{Background}

The latest global and Chinese cancer statistics show that the incidence of breast cancer in women is still ranked the first, and the morbidity trend is increasing[1-4]. The median survival time of advanced breast cancer from diagnosis is $2-3$ years. Only about $5-10 \%$ of patients can survive for more than 5 years[5].

According to the tumor status of the patients, various treatments are combined to formulate an effective comprehensive treatment program[6]. For the patients with strong expression (3+) of Her-2 in immunohistochemistry (IHC) report, or expression (2+) by IHC and confirmed to be positive by FISH, targeted therapy is an internationally accepted method. Currently available HER2-targeting drugs have vastly improved outcomes in patients with HER2/Her-2 positive status [7]. Accurate and sensitive detection of this targeted factor plays a critical role in screening patients for targeted treatment. However, the IHC/FISH technology, which is mainly used to assess the Her-2 status on tissue, cannot reveal the real gene status in tumors of the patient due to the potentially inconsistent HER2 gene status between primary and metastatic tumors.

Circulating tumor cells (CTCs), a liquid biopsy specimen, are highly active tumor cells that detach from solid tumors and enter the peripheral blood. It has been confirmed that CTCs derived from highly invasive tumors and are associated with tumor metastasis[8]. Lots of previous studies have shown that CTCs are 
significantly correlated with the prognosis and survival of multiple solid tumors, such as metastatic breast cancer[9], prostate cancer[10], and colorectal cancer[11]. Emerging studies also showed that CTC can be a potential biomarker for patient stratification and targeted therapy selection[12]. It is difficult to obtain multiple tumor tissue samples in advanced and metastasis cancer. In this situation, CTCs could be an ideal source of cancer cells for genomic analysis. Tumor tissue sample analysis is usually unable to detect all the genetic variants present in the heterogeneous tumors such as breast cancer[13]. Evidence has pointed out that molecular profiling of CTCs provides a better representation of tumor diversity than a single biopsy[14].

Multiple gene variations in single CTC can be investigated through whole genome sequencing (WGS) based on the next generation sequencing (NGS) technology $[15,16]$. With the development of CTC isolation and NGS technology, our understanding of intra-tumor heterogeneity has significantly improved, and the individualized treatment and precise treatment of tumors are further advanced.

In this study, CTC single cell sequencing was performed and the WGS data of CTCs was compared with tumor pathologic results of 3 patients with breast cancer. We evaluated the tumor heterogeneity, optimized the treatment decision of these patients, and achieved significant clinical results.

\section{Results}

\section{CTC enumeration of breast cancer patients}

A total of 24 CTCs with chromosome aneuploidy were enriched and identified in all three patients (Table 1, Fig. 1). The number of CTCs for the 3 patients was 10,7 and 7 cells $/ 7.5 \mathrm{ml}$ blood respectively. All these cells were negative for Her-2 expression in the membrane surface. One single CTC for every patient was retrieved by micro dissection for the WGS analysis. It was pentaploid cell that collected from patient ID1 and patient ID3, and tetraploid cell from patient ID2. Paired white blood cells were used as control.

Table 1

CTC number assessed for each patient based on chromosome aneuploidy

\begin{tabular}{|llllll|}
\hline Patient ID & \multicolumn{2}{l}{ Number of singles for CEP8 in CTCs } & \multirow{2}{*}{ CTC with Her2 expression } & Total \\
\cline { 2 - 4 } & Triploid & Tetraploid & Pentaploid and above & \\
\hline 1 & 7 & 1 & 2 & 0 & 10 \\
\hline 2 & 6 & 1 & 0 & 0 & 7 \\
\hline $3^{\text {bT }}$ & 6 & 0 & 1 & 0 & 7 \\
\hline $3^{\text {aT }}$ & 0 & 0 & 0 & 0 & 0 \\
\hline bT: before treatment; ${ }^{\text {aT: }}$ after treatment & & \\
\hline
\end{tabular}




\section{Somatic mutation and CNV in CTCs}

We identified 2,934, 497, and 2,901 mutations in the CTCs of the three patients, respectively. About $20 \%$ of these mutations were recorded in the COSMIC database (Table 2). Besides, TP53 R248W was identified in patient ID3, which was identified by the 57-panel in the ctDNA of patient ID3 as well.

HER2 Amplification was observed in all the 3 patients (Table 2). Besides, CNV gains of other 2 drugrelated genes (FGFR1 and FGFR2) were revealed in patient ID3.

Table 2

DNA status in CTCs and ctDNA of patients with breast cancer

\begin{tabular}{|c|c|c|c|c|c|}
\hline \multirow[t]{2}{*}{ Sample } & \multicolumn{3}{|c|}{ DNA of CTCs } & \multicolumn{2}{|c|}{ ctDNA in patient ID3 } \\
\hline & ID 1 & ID2 & ID3 & before TM* & after TM* \\
\hline \multicolumn{6}{|l|}{ Mutation } \\
\hline Total recorded & 2934 & 497 & 2901 & & \\
\hline Cosmic recorded & 583 & 95 & 538 & & \\
\hline Breast cancer-related & 30 & 8 & 39 & & \\
\hline Chromosome 17 & 174 & 35 & 201 & & \\
\hline TP53 mutation & 0 & 0 & R248W & R248W & - \\
\hline \multicolumn{6}{|l|}{ CNV } \\
\hline ERBB2 & 3 & 4 & 8 & - & - \\
\hline FGFR1 & 2 & 2 & 5 & 3 & - \\
\hline FGFR2 & 2 & 2 & 7 & - & - \\
\hline
\end{tabular}

\section{Correlation between HER2 status and patients' treatment}

Patient ID1 with Her-2 protein expression in the tumor by IHC (Fig. 2a) showed HER2 gene amplification according to the WGS analysis of CTC (Table 2). The first diagnosis of nonspecific invasive breast cancer was made in Oct 2017. After a mastectomy, she received chemotherapy combined with molecular targeted therapy TCH (Paclitaxel-Carboplatin-Trastuzumab) every 21 days for 6 cycles. Trastuzumab continued for a year. Endocrine therapy is ongoing. Disease free survival (DFS) reached 18 months after followed up to May 2019. (Fig. 3).

Patient ID2 with Her-2 protein expression in the tumor by IHC (Fig. 2a) showed HER2 gene amplification in the WGS analysis of CTC (Table 2). Nonspecific invasive breast cancer was diagnosed in Nov 2017. After a mastectomy, she was treated with anthracycline plus cyclophosphamide (AC) every 21 days for 4 
cycles followed docetaxel combined with trastuzumab $(\mathrm{TH})$ for 4 cycles. Trastuzumab continued for a year. Endocrine therapy is ongoing. Disease free survival (DFS) reached 18 months after followed up to May 2019. (Fig. 3).

Very little expression (1+) of Her-2 protein was found in primary and liver metastasis tumor of Patient ID3 by IHC test, which was defined as Her-2 negative (Fig. 2a). Subsequently, Her-2 negative was demonstrated in both tumors by FISH test (Fig. 2b). However, amplification in HER2 gene according to the WGS analysis of CTC was observed in this patient (Table 2). Diagnosis of breast infiltrating ductal cancer was made in Nov 2015. Neoadjuvant chemotherapy with docetaxel, epirubicin and cyclophosphamide (TEC) was performed before the mastectomy and continued adjuvant chemotherapy and radiotherapy after the surgery. After that treatment, the patient was treated with endocrine therapy. In Oct 2017, pleural effusion, peritoneal effusion and liver multiple occupancies were observed in the patient. No cancer cell was found in the pleural effusion, but invasive adenocarcinoma was found in the biopsy of liver tissue, suggesting the liver metastasis. Her-2 negative in the metastasis tissue was confirmed by both $\mathrm{IHC}$ and FISH tests. After a treatment of paclitaxel combined with capecitabine, the liver metastases were reduced, and some tumor cells were identified in the pleural effusion. In Mar 2018, pleural effusion increased a lot and trastuzumab was added into the chemotherapy. Two month later, the pleural effusion disappeared, and tumor partial remission was much more obviously observed compared to the effect of chemotherapy only. Endocrine therapy combined trastuzumab is ongoing. A 18-month progression-free survival (PFS) was recorded for Patient ID3 until the manuscript was finished (Figs. 3 and 4).

\section{Changes of CTC and ctDNA in patient ID3 before and after the treatment}

CTCs and mutations of ctDNA (57 gene panel) were detected at first diagnosis and after the trastuzumab related treatment in patient ID3 (Fig. 4). At the first diagnosis, 7 CTCs/7.5 ml blood were identified, a variation of TP53 R248W and amplification of FGFR1 were observed in the plasma. After the trastuzumab related treatment, neither CTC in blood nor gene alternation in plasma was found in this patient (Table 2, Figs. 3 and 4).

\section{Discussion}

Breast cancer is a heterogeneous disease with high incidence, easy to recur and metastasis, which leads to that accurate diagnosis and treatment is still a hot and difficult point of research [17]. The intra-tumor heterogeneity of breast cancer is manifested in spatial and temporal, and individual tumors in one patient have different subpopulations of cancer cells in distant regions [18]. Circulating tumor cells (CTCs) have the same characteristics of primary or metastatic lesions. Some studies $[17,19,20]$ have proved that the tumor genome heterogeneity and micrometastase can be found by whole genome sequencing of CTC, and it is feasible to conduct tumor drug guidance according to the characteristics of CTC genome. In our study, genomic HER2 characteristics of CTC in Patient ID1 and Patient ID2 were consistent with tissue results by IHC tests, and targeted therapy was in line with expectations. HER2 gene status of CTC in 
Patient ID3 is not consistent with tissue results by either IHC or FISH tests. Both the little expression (1+) of Her-2 protein expression and the negative result of FISH were not support the Her-2 targeted therapy. However, Her-2 targeted therapy is effective for this patients after the failure of the first treatment, which demonstrated that genomic charaterizations of CTC can conduct the drug guidance of tumors.

Key driver mutations such as mutations of TP53 and PIK3CA, amplifications of MYC, CCND1, and HER2 usually take place in primary tumor cells $[21,22]$. Some gene alternation presented by WGS analysis of CTC, like CNV gains of HER2 gene in patient ID1 and ID2 consistent with the result of IHC detection for tissue, are potentially come from the primary tumors. Additional driver mutations or amplifications during the tumor progression may lead to further clonal diversity in primary or metastasis tumors and treatment resistance [23]. Numerous studies have shown that discordance rates of HER2 between primary and recurrent or metastatic tumors is $8-16 \%$ respectively [24-26]. In our study, CNV gains of HER2 gene was identified in the DNA of CTC but Her-2 protein expression was not observed in primary and liver metastasis tumor, which implied that HER2 CNV gained in another metastatic tumor that is not discovered at that moment. What' s more, no HER2 alternation was presented in ctDNA, which implied that the potential metastasis was too tiny to be identified clinically. Although increased pleural effusion suggested pulmonary metastasis, no tumor cell was found in pleural effusion sample. Heterogeneity in tumors poses a severe challenge to the diagnosis and prognosis of diseases. The heterogeneity of breast cancer is the main cause of many treatment failures. For the patient ID3, pleural effusion was still in the state of disease progression after chemotherapy. However, this pleural abnormality was released, enumeration of CTC and the burden of ctDNA in patient ID3 were decreased after the original regimen combined with trastuzumab treatment. Trastuzumab is an effective target drug for HER2-positive breast cancer $[27,28]$. Therefore, we speculate the existence of micrometastasis in the lung but not clinically detected. HER2-positive CTCs may be associated with lung metastasis, which ultimately leads to the ineffectiveness of previous chemotherapy.

In the investigation of patient ID3, no abnormal of HER2 was observed in the tumor tissues detected by IHC and FISH, membrane expression checking of CTC, or ctDNA sequencing. Only the WGS analysis of CTC revealed CNV gains of the HER2 gene. HER2 detection on tissues is commonly used as a criterion for targeting therapy in the clinic. However, due to the heterogeneity of tumors and the defects of detection techniques, the detection results are inaccurate. Likewise, the detection of Her-2 protein expression on CTC cell membranes also has problems considering the epithelial-mesenchymal transition [29, 30]. The ctDNA assay is limited by the design of the assay panel, and the tumor signal is diluted and eventually prone to false negative [31, 32]. Previous studies pointed that when traditional tissue biopsies are difficult to obtain, CTCs sequencing may provide an alternative method for comprehensive genome studies to analyze tumor heterogeneity and obtain optimal targets for therapeutic $[14,20]$, which was consistent with our results.

\section{Conclusions}


This is the first time we have discovered an advanced breast cancer patient with a HER2 gene amplification in the single CTC by WGS but not histologically expressed in the tumor tissue. CTC sequencing makes us further understand the heterogeneity of breast cancer, permits the non-invasive and repeated accurate monitoring of therapeutic response and tumor progression, helps us to make therapeutic decisions and predict the outcome, which ultimately achieves personalized molecularly guided cancer treatment. Since this is a retrospective study with a small number of cases, large sample prospective control study is necessary in the future. The single cell sequencing in this study was performed two years ago, for which the quality control of CTC, single cell separation and whole genome sequencing need improving. In the past two years, the development of single cell technology has made rapid progress, which can analyze a large number of samples, increase the detection accuracy, reduce the economic cost, and improve the clinical application.

\section{Methods}

\section{Patients and sample collection}

Patients enrolled in this study were diagnosed at Shanghai Pudong Hospital. Our study was approved by the Shanghai Pudong Hospital Ethics Committee (No. W2001). Written informed consent were obtained to allow the sample collection and data analysis for research purposes. For CTC analysis, $7.5 \mathrm{ml}$ peripheral blood was drawn from patients into ACD anti-coagulant tube. For ctDNA analysis, another $10 \mathrm{ml}$ peripheral blood was collected into an EDTA anti-coagulant tube. Clinical characterizations of these patients were shown in supplementary table1.

\section{Investigation of CTCs}

Circulating tumor cell (CTC) detection was carried out by CTC detection kit (Majorbio, China). Briefly, this method combines subtraction enrichment, immunofluorescence staining and chromosome in situ hybridization to achieve the separation and identification of CTC [33]. Firstly, plasma was removed by centrifugation, and red blood cells were removed by density gradient centrifugation. White blood cells were specifically removed by antibody-coated magnetic beads, and remaining cells including CTCs were coated on a slide for subsequent cell identification. Secondly, cells on the slide were stained with multitumor immunofluorescence markers CD45 and Her-2. The centromere of chromosome 8 (CEP8) was detected by fluorescence in situ hybridization. Finally, CTCs were identified according to the criteria of CD45-, DAPI+, and CEP8 $\geq 3$. The expression of Her-2 on the surface of CTC was also recorded.

\section{Whole genome amplification and sequencing of single CTC}

For each patient, a single CTC was collected into a tube by microdissection performed on a PALM MicroBeam instrument (Zeiss) for subsequent CTC single cell whole genome amplification (WGA). Singlecell WGA was performed using the single-cell whole genome amplification kit (Yikon Genomics, China) which is based on the MALBAC method [34]. The amplified DNA product was assessed by a Qubit ${ }^{\circledR}$ dsDNA HS Assay kit in a Qubit 3.0 Fluorometer (Life Technologies, USA), evaluated the molecular weight 
on $1 \%$ agarose gel electrophoresis, and checked the genomic integrity of the amplified DNA product by quantitative PCR (qPCR) with eight randomly selected loci was performed. DNA samples with a total mass more than $2 \mu \mathrm{g}$, and DNA fragment range $300 \mathrm{bp}$ to $2000 \mathrm{bp}$ were considered to meet the further sequencing criteria.

Library preparation was performed according to the SureSelectXT Illumina Paired-End Sequencing Library protocol. Library quality was assessed by 2100 Bioanalyzer and qPCR with TBS380 picogreen (Invitrogen,USA). Next Seq CN500 High-throughput Sequencer (Illumina) was used for whole-genome sequencing of the captured single cells.

\section{ctDNA analysis of the metastatic patient}

In this study, plasma DNA of patient ID3 with metastasis breast cancer was extracted for ctDNA analysis. A total of 67 cancer-related genes including 57 drugs related genes, 2 endocrine therapy related genes, and 9 chemotherapy related genes. Point mutations, small fragment insertions and deletions, copy number variants, and fusions of these genes were detected. Details of the gene panel are shown in Supplementary Table 2.

\section{Abbreviations}

CTCs: Circulating tumor cells; WGS:whole genome sequencing; IHC:immunohistochemistry; CEP8:centromere of chromosome 8; NGS:next generation sequencing; qPCR:quantitative PCR; DFS:Disease free survival; PFS:progression-free survival.

\section{Declarations}

\section{Ethical approval and consent to participate:}

All procedures performed in studies involving human participants were in accordance with the ethical standards of Shanghai Pudong Hospital Ethics Committee (NO. W2001) and with the 1964 Helsinki declaration and its later amendments or comparable ethical standards. Written informed consent was obtained from individual participants.

\section{Consent for publication:}

Not applicable.

\section{Availability of data and material:}

Not applicable.

\section{Competing interest:}

The authors declare that they have no conflicts of interest. 


\section{Funding:}

This work was funded by Key Specialty Construction Project of Pudong Health and Family Planning Commission of Shanghai: PWZzk2017-32, Talents Training Program of Pudong Hospital affiliated to Fudan University: RHJJ 2018-01, Subject of Advanced talents in Shanghai area YJRCJJ201701, and National Natural Science Foundation of China (81672593₫81272899) .

\section{Authors' contributions:}

Yongping Li, Hao Yuan, Bin Zhang were equal contribute to this article. All authors were involved in drafting and finalizing the report.

\section{Acknowledgements:}

We thank the patients, physicians, nurses, and data managers who participated in the trial.

\section{References}

1. Bray F, Ferlay J, Soerjomataram I, Siegel RL, Torre LA, Jemal A. Global cancer statistics 2018: GLOBOCAN estimates of incidence and mortality worldwide for 36 cancers in 185 countries. CA Cancer J Clin. 2018;68(6):394-424.

2. Siegel RL, Miller KD, Jemal A. Cancer statistics, 2018. CA Cancer J Clin. 2018;68(1):7-30.

3. Chen W, Zheng R, Zhang S, Zeng H, Xia C, Zuo T, Yang Z, Zou X, He J. Cancer incidence and mortality in China, 2013. Cancer Lett. 2017;401:63-71.

4. Zheng R, Zeng H, Zhang S, Chen W. Estimates of cancer incidence and mortality in China, 2013. Chin J Cancer. 2017;36(1):66.

5. Huober J, Thurlimann B. The Role of Combination Chemotherapy in the Treatment of Patients with Metastatic Breast Cancer. Breast Care (Basel). 2009;4(6):367-72.

6. Curigliano G, Burstein HJ, Gnant EPW, Dubsky M, Loibl P, Colleoni S, Regan M, Piccart-Gebhart MM, Senn M. HJ et al: De-escalating and escalating treatments for early-stage breast cancer: the St. Gallen International Expert Consensus Conference on the Primary Therapy of Early Breast Cancer 2017. Ann Oncol 2019.

7. Bartsch R, Bergen E. ASCO 2018: highlights in HER2-positive metastatic breast cancer. Memo. 2018;11(4):280-3.

8. Lianidou ES, Strati A, Fau - Markou A, Markou A. Circulating tumor cells as promising novel biomarkers in solid cancers. Crit Rev Clin Lab Sci. 2014;51(3):160-71.

9. Cristofanilli M, Budd GT, Ellis MJ, Stopeck A, Matera J, Miller MC, Reuben JM, Doyle GV, Allard WJ, Terstappen LW, et al. Circulating tumor cells, disease progression, and survival in metastatic breast cancer. N Engl J Med. 2004;351(8):781-91. 
10. de Bono JS, Scher HI, Montgomery RB, Parker C, Miller MC, Tissing H, Doyle GV, Terstappen LW, Pienta KJ, Raghavan D. Circulating tumor cells predict survival benefit from treatment in metastatic castration-resistant prostate cancer. Clin Cancer Res. 2008;14(19):6302-9.

11. Cohen SJ, Punt CJ, lannotti N, Saidman BH, Sabbath KD, Gabrail NY, Picus J, Morse M, Mitchell E, Miller MC, et al. Relationship of circulating tumor cells to tumor response, progression-free survival, and overall survival in patients with metastatic colorectal cancer. Journal of clinical oncology: official journal of the American Society of Clinical Oncology. 2008;26(19):3213-21.

12. Lee JS, Magbanua MJM, Park JW. Circulating tumor cells in breast cancer: applications in personalized medicine. Breast Cancer Res Treat. 2016;160(3):411-24.

13. Kim C, Paik S. Gene-expression-based prognostic assays for breast cancer. Nat Rev Clin Oncol. 2010;7(6):340-7.

14. Lohr JG, Adalsteinsson VA, Cibulskis K, Choudhury AD, Rosenberg M, Cruz-Gordillo P, Francis JM, Zhang C-Z, Shalek AK, Satija R, et al. Whole-exome sequencing of circulating tumor cells provides a window into metastatic prostate cancer. Nat Biotechnol. 2014;32(5):479-84.

15. Katsios C, Roukos DH. Individual genomes and personalized medicine: life diversity and complexity. Per Med. 2010;7(4):347-50.

16. Gulbahce N, Magbanua MJM, Chin R, Agarwal MR, Luo X, Liu J, Hayden DM, Mao Q, Ciotlos S, Li Z, et al. Quantitative Whole Genome Sequencing of Circulating Tumor Cells Enables Personalized Combination Therapy of Metastatic Cancer. Cancer Res. 2017;77(16):4530-41.

17. Joseph C, Papadaki A, Althobiti M, Alsaleem M, Aleskandarany MA, Rakha EA. Breast cancer intratumour heterogeneity: current status and clinical implications. Histopathology. 2018;73(5):71731.

18. Navin N, Krasnitz A, Rodgers L, Cook K, Meth J, Kendall J, Riggs M, Eberling Y, Troge J, Grubor V, et al. Inferring tumor progression from genomic heterogeneity. Genome Res. 2010;20(1):68-80.

19. Brouwer A, De Laere B, Peeters D, Peeters M, Salgado R, Dirix L, Van Laere S. Evaluation and consequences of heterogeneity in the circulating tumor cell compartment. Oncotarget. 2016;7(30):48625-43.

20. Gulbahce N, Magbanua MJM, Chin R, Agarwal MR, Luo X, Liu J, Hayden DM, Mao Q, Ciotlos S, Li Z, et al. Quantitative Whole Genome Sequencing of Circulating Tumor Cells Enables Personalized Combination Therapy of Metastatic Cancer. Can Res. 2017;77(16):4530-41.

21. Stephens PJ, Tarpey PS, Davies H, Van Loo P, Greenman C, Wedge DC, Nik-Zainal S, Martin S, Varela I, Bignell GR, et al. The landscape of cancer genes and mutational processes in breast cancer. Nature. 2012;486(7403):400-4.

22. Nik-Zainal S, Van Loo P, Wedge DC, Alexandrov LB, Greenman CD, Lau KW, Raine K, Jones D, Marshall J, Ramakrishna M, et al. The life history of 21 breast cancers. Cell. 2012;149(5):994-1007.

23. Almendro V, Kim HJ, Cheng YK, Gonen M, Itzkovitz S, Argani P, van Oudenaarden A, Sukumar S, Michor F, Polyak K. Genetic and phenotypic diversity in breast tumor metastases. Cancer Res. 2014;74(5):1338-48. 
24. Amir E, Miller N, Geddie W, Freedman O, Kassam F, Simmons C, Oldfield M, Dranitsaris G, Tomlinson G, Laupacis A, et al. Prospective study evaluating the impact of tissue confirmation of metastatic disease in patients with breast cancer. J Clin Oncol. 2012;30(6):587-92.

25. Niikura N, Liu J, Hayashi N, Mittendorf EA, Gong Y, Palla SL, Tokuda Y, Gonzalez-Angulo AM, Hortobagyi GN, Ueno NT. Loss of human epidermal growth factor receptor 2 (HER2) expression in metastatic sites of HER2-overexpressing primary breast tumors. J Clin Oncol. 2012;30(6):593-9.

26. Lindström LS, Karlsson E, Wilking UM, Johansson U, Hartman J, Lidbrink EK, Hatschek T, Skoog L, Bergh J. Clinically Used Breast Cancer Markers Such As Estrogen Receptor, Progesterone Receptor, and Human Epidermal Growth Factor Receptor 2 Are Unstable Throughout Tumor Progression. J Clin Oncol. 2012;30(21):2601-8.

27. Singer CF, Balmaña J, Bürki N, Delaloge S, Filieri ME, Gerdes A-M, Grindedal EM, Han S, Johansson O, Kaufman B, et al. Genetic counselling and testing of susceptibility genes for therapeutic decisionmaking in breast cancer-an European consensus statement and expert recommendations. Eur J Cancer. 2019;106:54-60.

28. Howie LJ, Scher NS, Amiri-Kordestani L, Zhang L, King-Kallimanis BL, Choudhry Y, Schroeder J, Goldberg KB, Kluetz PG, Ibrahim A, et al: FDA Approval Summary: Pertuzumab for adjuvant treatment of HER2-positive early breast cancer. Clinical Cancer Research 2018:clincanres.3003.2018.

29. Guan X, Ma F, Li C, Wu S, Hu S, Huang J, Sun X, Wang J, Luo Y, Cai R, et al. The prognostic and therapeutic implications of circulating tumor cell phenotype detection based on epithelialmesenchymal transition markers in the first-line chemotherapy of HER2-negative metastatic breast cancer. Cancer Commun (Lond). 2019;39(1):1.

30. Papadaki MA, Stoupis G, Theodoropoulos PA, Mavroudis D, Georgoulias V, Agelaki S. Circulating tumor cells with stemness and epithelial-to-mesenchymal transition features are chemoresistant and predictive of poor outcome in metastatic breast cancer. Mol Cancer Ther 2018.

31. Clark TA, Chung JH, Kennedy M, Hughes JD, Chennagiri N, Lieber DS, Fendler B, Young L, Zhao M, Coyne M, et al. Analytical Validation of a Hybrid Capture-Based Next-Generation Sequencing Clinical Assay for Genomic Profiling of Cell-Free Circulating Tumor DNA. J Mol Diagn. 2018;20(5):686-702.

32. Lanman RB, Mortimer SA, Zill OA, Sebisanovic D, Lopez R, Blau S, Collisson EA, Divers SG, Hoon DS, Kopetz ES, et al. Analytical and Clinical Validation of a Digital Sequencing Panel for Quantitative, Highly Accurate Evaluation of Cell-Free Circulating Tumor DNA. PLoS One. 2015;10(10):e0140712.

33. Lin PP. Integrated EpCAM-independent subtraction enrichment and iFISH strategies to detect and classify disseminated and circulating tumors cells. Clin Transl Med. 2015;4(1):38.

34. Zong C, Lu S, Chapman AR, Xie XS. Genome-wide detection of single-nucleotide and copy-number variations of a single human cell. Science. 2012;338(6114):1622-6.

\section{Supplementary Information}

Tables S1 and S2 were not provided with this version of the manuscript. 
Additional file 1: Table S1. Clinical information and expression of tumor markers of the breast cancer patients.

Additional file 2: Table S2. Gene panel of ctDNA sequencing analysis.

\section{Figures}
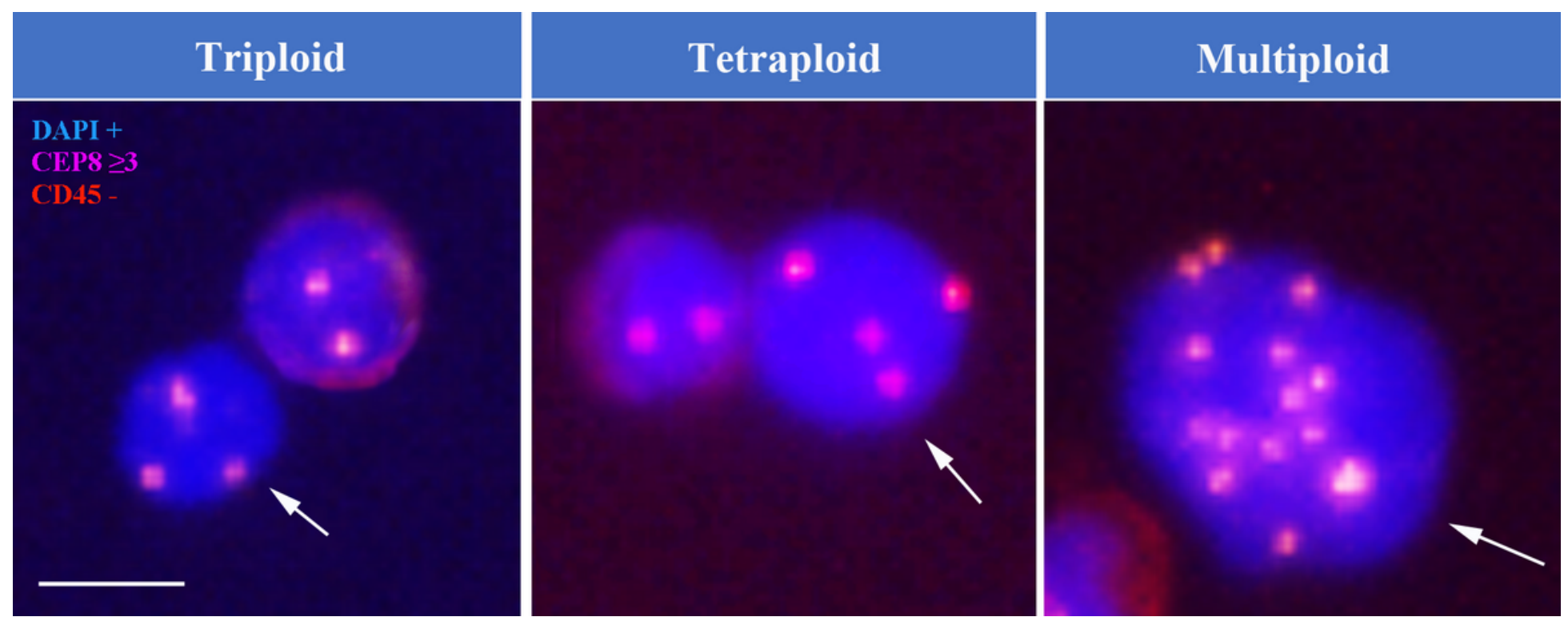

Figure 1

CTCs in patients with breast cancer. White arrows showed the identified CTCs. Scale bar is $10 \mu \mathrm{m}$. 

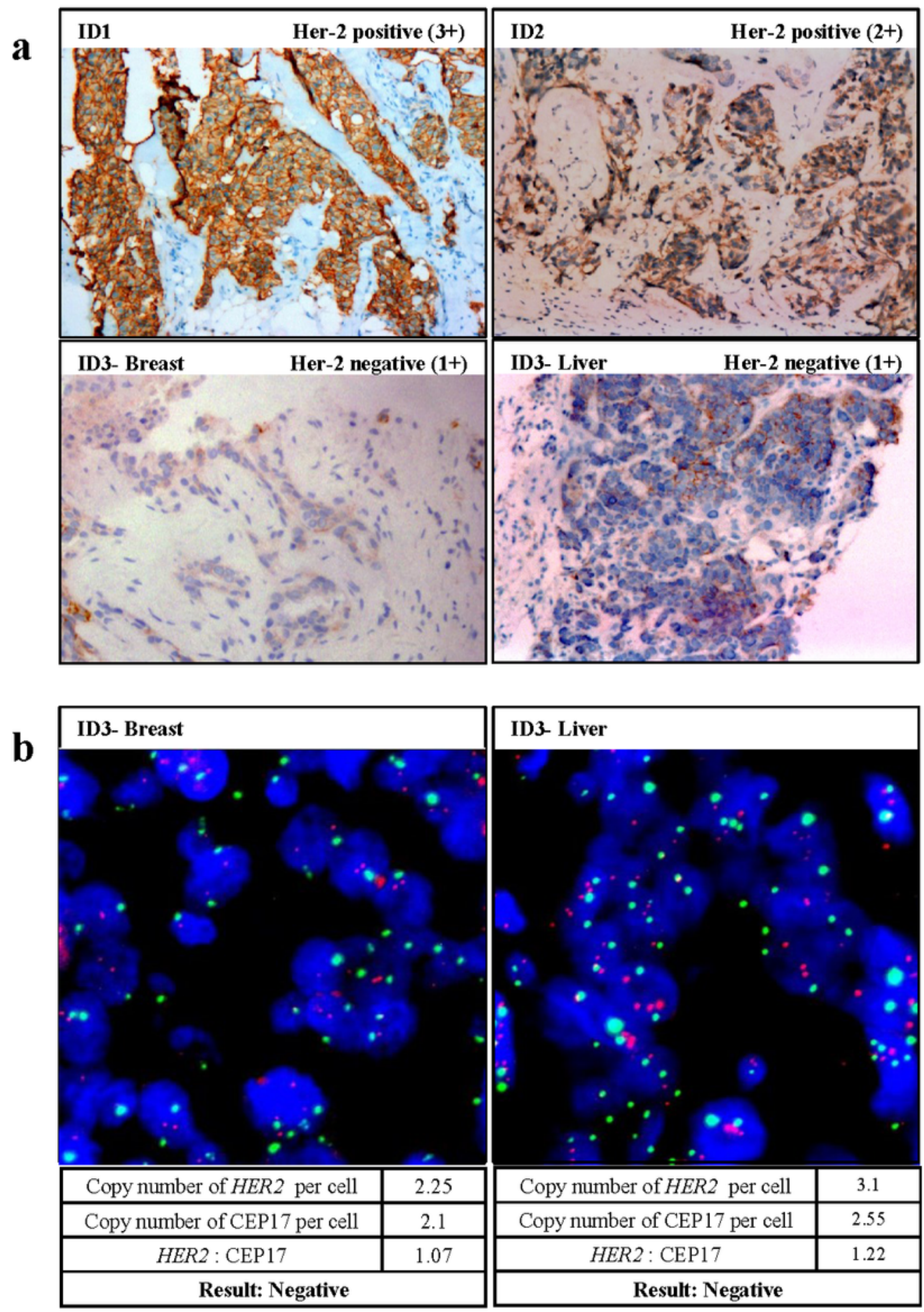

\section{Figure 2}

Her-2/HER2 status in the tissue of the 3 patients. a. The Her-2 protein expression in the primary tissue of patient ID1, ID2, and ID3, and in the liver metastasis tissue of patient ID3 were detected by IHC test. b. The copy number of HER2 gene in the primary and liver metastasis tissue of patient ID3 were detected by FISH test. 


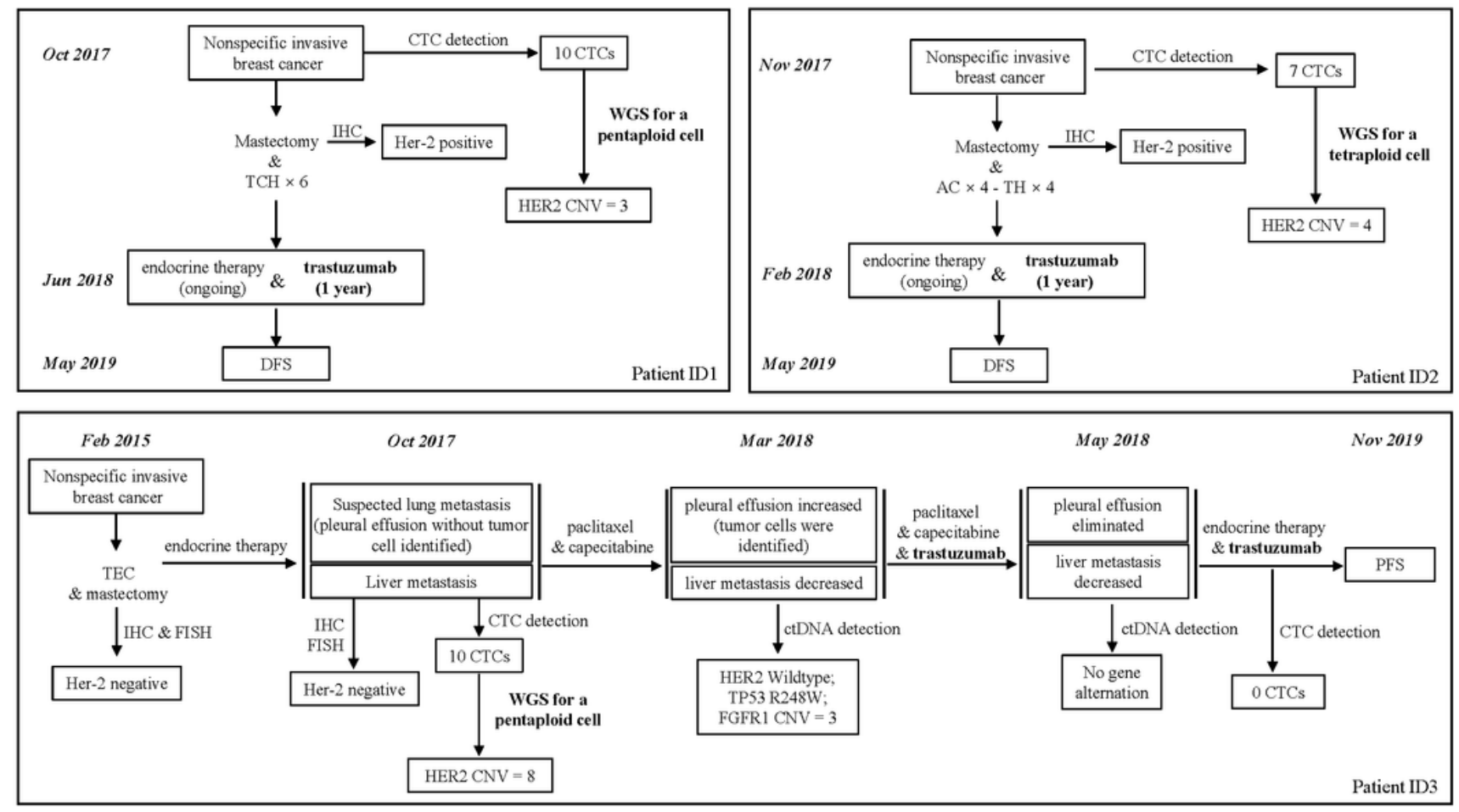

\section{Figure 3}

Clinical timelines of the 3 patients with breast cancer. The HER2 targeted drug trastuzumab were highlighted in bold type. DFS, disease-free survival; PFS, progression-free survival.

\begin{tabular}{|c|c|c|c|c|c|c|}
\hline Primary diagnosis & Tumor remission & Tumor metastasis & Tumor progression & Tumor remission & Tumor remission \\
Mastectomy \\
Endocrine therapy
\end{tabular}




\section{Figure 4}

Variations of imaging and molecular biomarker features in Patient ID3 during a series of medical treatments. Treatment process is showed in the top. Images of the lung (upper) and liver (lower) metastasis are listed in the middle. Variations of CTC (grey column) and ctDNA (black line for CNV of FGFR1; grey line for mutation of TP53 R248W) are presented in the bottom. 